$\Rightarrow$ T-CELL DEVELOPMENT

\title{
Life and death with the IAN family
}

Immune-associated nucleotide-binding proteins (IANs; also known as GIMAPs) constitute a family of GTP-binding proteins that are expressed by vertebrate immune cells. Until recently, little has been known about the function of these proteins. Now, a report in PLoS Biology shows that IAN1, IAN4 and IAN5 are crucial regulators of T-cell development in the thymus.

Developing $T$ cells must pass through a series of checkpoints in the thymus. These include positive selection and negative selection, both of which depend on T-cell receptor (TCR) signalling and which result, respectively, in the survival of thymocytes that express a functional TCR and in the apoptotic death of thymocytes that strongly recognize ligand. Yousuke Takahama and colleagues set out to study the molecular mechanisms that regulate these selection processes. When examining the transcriptional profile of mouse thymocytes with various developmental characteristics, they found that the IAN-family proteins IAN1 and IAN4 are strongly upregulated by thymocytes that have undergone positive selection (that is, in cells that have progressed from the double-positive (DP) to the single-positive stage of development). They confirmed this finding using quantitative realtime PCR and additionally showed that IAN5 is also upregulated at this stage. However, enforced overexpression of IAN1 (but not IAN4 or IAN5) at an early stage of development (in double-negative thymocytes) was found to result in apoptosis at the DP stage, whereas inhibition of endogenous expression of IAN4 or IAN5 (but not IAN1) blocked thymocytes from reaching or progressing past the positive-selection checkpoint.

So, IAN-family members are novel TCR-responsive proteins that crucially and differentially regulate the survival and death of thymocytes. In subsequent experiments, IAN1 was found to associate selectively with the proapoptotic BCL-2 (B-cell lymphoma 2)-family member $\mathrm{BAX}$ (BCL-2-associated $\mathrm{X}$ protein), whereas IAN4 and IAN5 were found to associate with anti-apoptotic $B C L-2$-family members, including $B C L-2$ and $B C L-X_{L}$. These findings indicate that IANs might exert their functions by relaying signals that regulate apoptosis from the TCR to BCL-2-family members, and this improves our understanding of the mechanism by which TCR signalling influences the fate of thymocytes.

Davina Dadley-Moore

ORIGINAL RESEARCH PAPER Nitta, T. et al. IAN family critically regulates survival and development of

T lymphocytes. PLoS Biol. 4, e103 (2006)
$\Rightarrow$ T CELLS

\section{De novo generation of IL-17-producing T cells}

The mechanism involved in the de novo differentiation of interleukin-17 (IL-17)-producing T cells from naive $\mathrm{CD}^{+} \mathrm{T}$ cells is not fully understood, but it has been suggested that IL-23 might be involved. Now, new research published in Immunity highlights a pivotal role for transforming growth factor- $\beta$ (TGF $\beta$ ), together with the pro-inflammatory cytokine IL- 6 , in the generation of these cells.

The naturally occurring $\mathrm{CD} 4{ }^{+} \mathrm{CD} 25^{+}$regulatory $\mathrm{T}\left(\mathrm{T}_{\mathrm{Reg}}\right)$-cell subset - which can prevent immune pathology through the suppression of potentially pathogenic $\mathrm{T}$ cells - suppress proliferation and IL-2 production by naive $\mathrm{CD} 4^{+} \mathrm{T}$ cells in vitro. However, Stockinger and colleagues show that in the presence of an inflammatory stimulus, such as lipopolysaccharide (LPS), $\mathrm{T}_{\mathrm{Reg}}$ cells do not suppress T-cell proliferation but still retain the ability to suppress the production of IL-2 and also interferon- $\gamma(\mathrm{IFN} \gamma)$. Interestingly, coculture of $\mathrm{T}_{\mathrm{Reg}}$ cells with naive $\mathrm{CD} 4^{+}$ $\mathrm{T}$ cells, in the presence of dendritic cells (DCs) and LPS, results in the induction of IL-17-producing T cells.

TGF $\beta$, an immunosuppressive cytokine that is produced by various cell types including $\mathrm{T}_{\mathrm{Reg}}$ cells, was

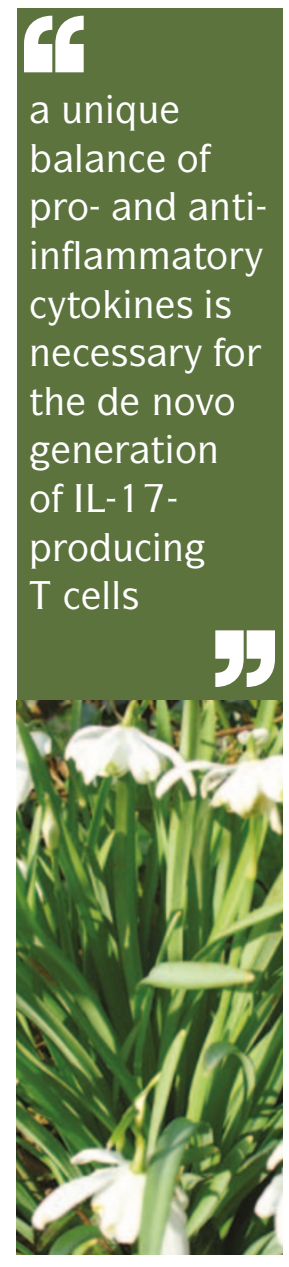

identified as the key component in this differentiation process. Blocking TGF $\beta$, but not the $\mathrm{T}_{\mathrm{Reg}}$-cell-associated cytokine IL-10, with neutralizing antibodies suppressed the generation of IL-17-producing T cells. The $\mathrm{T}_{\text {Reg }}$ cells could also be replaced in the co-culture by addition of TGF $\beta$, highlighting the crucial role of this cytokine in the differentiation of IL-7-producing T cells.

This study also showed that in addition to TGF $\beta$, a DC-derived factor is required, as the generation of IL-17-producing T cells did not occur in the absence of DCs. The authors identified the cytokine IL-6 as this essential mediator. Blocking of IL-6 suppressed the development of IL-17-producing T cells, whereas tumour-necrosis factor (TNF) and IL-1 $\beta$ further augmented this differentiation. Interestingly, inhibition of IL-23 did not affect the differentiation of these cells, indicating that this cytokine probably does not have a role in the de novo generation of IL-17-producing T cells, although it is an important factor for their survival and expansion.

Co-culture of naive $\mathrm{CD} 4^{+} \mathrm{T}$ cells with DCs and LPS in the presence of antibodies specific for the
T helper $1\left(\mathrm{~T}_{\mathrm{H}} 1\right)$-associated cytokines IL-12, IL-23 and IFN $\gamma$ and the $\mathrm{T}_{\mathrm{H}} 2$-associated cytokine IL-4, resulted in the differentiation of IL-17-producing T cells. Inclusion of TGF $\beta$-specific antibodies inhibited this differentiation, indicating that, in addition to TGF $\beta$ produced by $\mathrm{T}_{\mathrm{Reg}}$ cells, LPS-stimulated DCs can produce TGF $\beta$, albeit in amounts that cannot support IL-17-producing T-cell differentiation without further blockade of cytokines that promote the differentiation of the other T-cell subsets.

Therefore, TGF $\beta$, together with IL-6, is essential for the de novo differentiation of IL-17-producing T cells from naive $\mathrm{CD}^{+} \mathrm{T}$ cells. It seems that, in addition to the suppressive role of TGF $\beta$ on $\mathrm{T}_{\mathrm{H}} 1$, and $\mathrm{T}_{\mathrm{H}} 2$-cell differentiation, this cytokine might also have a more direct role in the differentiation of IL-17-producing T cells. So, this study shows that a unique balance of pro- and anti-inflammatory cytokines is necessary for the de novo generation of IL-17-producing T cells.

Olive Leavy

ORIGINAL RESEARCH PAPER Veldhoen, M., Hocking, R. J., Atkins, C. J., Locksley, R. M. \& Stockinger, B. TGF $\beta$ in the context of an inflammatory cytokine milieu supports de novo differentiation of IL-17-producing T cells. Immunity 24, 179-189 (2006) FUTHER READING Dong, C. Diversification of T-helper-cell lineages: finding the family root of IL-17-producing cells. Nature Rev. Immunol. 17 Mar 2006 (doi:10.1038/nri1807) 